\title{
Développement de la méthode de Monte Carlo pour le calcul des interactions et du transport électrique dans les semi-conducteurs ternaires
}

\section{Abdelkader HAMDOUNE*, Benyounes BOUAZZA, Ahlem Guen-BOUAZZA, Abdelhafid LALLAM et Nasr-Eddine CHABANE-SARI}

Unité de recherche des matériaux et des énergies renouvelables, Faculté des sciences de l'ingénieur, Université Abou-bekr Belkaid. Tlemcen 13000. Algérie.

(Reçu le 04 Novembre 2006, accepté le 11 Avril 2007)

*Correspondance,courriel : d_hamdoune@yahoo.fr

\section{Résumé}

Dans ce papier, nous montrons en premier lieu l'intérêt des hétérostructures, puis la nécessité d'utiliser une méthode numérique et notamment celle de Monte Carlo, pour calculer le transport électrique dans les semi-conducteurs. Nous justifions aussi la composition de notre semi-conducteur ternaire AlxGal-xAs. Ensuite, nous donnons le principe et la mise en œuvre de la méthode que nous avons adaptée à notre cas, en essayant de nous approcher de la réalité. Nous appliquons enfin cette méthode pour calculer les interactions et le transport électrique dans notre composé.

Mots-clés : Monte Carlo, les interactions, piézoélectriques, acoustiques, intravallées, intervallées, optiques polaires.

\section{Abstract \\ Development of the Monte Carlo method for the calculation of the interactions and electric transport in the ternary semiconductors}

In this paper; we show the interest of the heterostructures initially, then the need for using a numerical method and in particular that of Monte Carlo, to calculate electric transport in the semiconductors. We justify also the composition of our ternary semiconductor AlxGal-xAs. Afterwards; we give the principle and the implementation of 
the method which we adapted to our case, while trying to approach us reality. We apply finally this method to calculate the interactions and electric transport in our compound.

\section{Keywords : Monte Carlo, piezoelectric, acoustic, intravalley, intervalley, polar optical interactions.}

\section{Introduction}

Des semi-conducteurs binaires III-V comme l'arséniure de gallium (GaAs), l'antimoniure de gallium (GaSb), le phosphure d'indium (InP), l'arséniure d'indium (InAs), l'arséniure d'aluminium (AIAs), le nitrure d'aluminium (AIN), le nitrure de gallium (GaN), le nitrure d'indium (InN), et leurs alliages ternaires et quaternaires possèdent des propriétés très intéressantes pour des applications en hyperfréquence.

Les hétérostructures supplantent les homostructures, aussi bien pour les transistors bipolaires que pour ceux à effet de champ. Elles ont pu être insérées dans la fabrication des composants actifs, grâce au progrès réalisé dans les techniques d'épitaxie. La particularité de ces structures résulte de leur capacité à contrôler le flux et la distribution des électrons et des trous à travers les décalages de bandes. Certains transistors sont constitués d'alliages ternaires déposés en couche mince, par épitaxie, sur un substrat binaire. Leur fonctionnement repose sur l'existence d'hétérojonctions semi-conducteur / semi-conducteur.

Le phénomène de transport dans ces semi-conducteurs résulte du comportement des électrons de la bande de conduction et la bande interdite. Une étude analytique du problème nécessite la connaissance de la fonction de distribution de l'énergie des électrons, une fonction obtenue en résolvant l'équation de Boltzmann. Cette dernière, aux dérivées partielles, n'admet de solutions analytiques que dans un nombre de cas limité.

Pour simuler le phénomène de transport électrique dans les semi-conducteurs [1,2], on utilise des méthodes numériques, et le plus fréquemment celle de Monte Carlo écrite en langage Fortran. Ce langage n'est pas nécessairement le meilleur en programmation scientifique, mais il est certainement le plus répandu : les programmes et sousprogrammes existants et relatifs à cette méthode, sont en Fortran [3-5].

Dans notre travail, nous essayons d'adapter cette méthode pour l'étude des semiconducteurs ternaires. Nous l'appliquons précisément pour le ternaire AlxGal-xAs déposé sur InP, nous y calculons les différentes interactions, les vitesses de dérive en régime transitoire dans toutes les vallées, ainsi que les vitesses de dérive moyennes. 
Lorsqu'on incorpore de l'aluminium dans GaAs, il en résulte une variation du paramètre de maille "a" du composé. En première approximation, ce paramètre varie linéairement avec le taux d'aluminium. Pour obtenir un bon accord de maille entre AlxGal-xAs et InP (GaAs), il faut que x soit égal à 0.15 , autrement dit, on doit incorporer $15 \%$ d'aluminium. Nous faisons alors le calcul pour l'alliage Al0.15 Ga085As.

\section{Principe et mise en œuvre de la méthode}

Le logiciel de la méthode permet de remplir deux fonctions essentielles. La première est consacrée au calcul des probabilités à partir des expressions usuelles. La deuxième fonction est destinée à la détermination des grandeurs instantanées définies sur un ensemble d'électrons (l'énergie, la vitesse, la position) par la procédure "Self Scattering II [2] pour laquelle, les durées de vol libre sont distribuées pour chaque électron.

La mise en œuvre a été adoptée initialement par Kurosawa [6], puis améliorée par d'autres auteurs [7-10]. Elle est basée sur un processus de tirage au sort à partir de lois de probabilités d'interactions subies par les porteurs durant leur mouvement dans le composé $[11,12]$.

Considérons un électron d'énergie $\varepsilon(t)$ et de vecteur d'onde $k(t)$, placé en $r(t)$ où règne un champ électrique $E(r, t)$. Sous l'action de $E$, les échanges d'énergie et d'impulsion de l'électron avec le réseau cristallin, et la déviation de sa trajectoire par les impuretés, vont modifier son énergie, son vecteur d'onde et sa position.

En appliquant les lois de la mécanique et de l'électrodynamique, nous pouvons déterminer théoriquement le comportement de chaque électron dans le temps et dans l'espace des vecteurs d'ondes. Mais, une telle approche, compte tenu de la très grande densité d'atomes dans les cristaux, est irréaliste avec ces moyens de calcul [13]. Afin de rendre le problème plus réaliste :

a)- Nous faisons une étude statistique des échanges d'énergie possibles, entre les électrons, les modes de vibrations du réseau et les impuretés, ce qui nous permet de calculer la probabilité de ces interactions et leur action sur l'énergie et le vecteur d'onde de l'électron.

Chaque processus de collision a une densité $s\left(\vec{k}, \overrightarrow{k^{\prime}}\right)$ qui caractérise la probabilité pour qu'un électron passe de l'état défini par le vecteur d'onde $\vec{k}$ à un autre état défini par le vecteur d'onde $\vec{k}^{\prime}$, par unité de temps. La probabilité pour qu'un électron, se trouvant dans l'état $k$, ait une interaction par unité de temps, est donnée par l'équation (I)[5]: 


$$
\lambda(k(t))=\int_{\substack{\text { sur tous les états } \\ k^{\prime} \text { possibles }}} s\left(\vec{k}, \vec{k}^{\prime}\right) d k^{\prime}
$$

b)- Nous supposons que ces interactions sont instantanées, les électrons sont alors déplacés en vol libre sous le seul effet du champ électrique, entre deux chocs. La probabilité d'avoir un vol libre de durée $\Delta t$, suivi d'un choc, est donnée par l'équation (2) [11] :

$$
p(\Delta t)=1-\exp \left[-\int_{0}^{\Delta t} \lambda(k(t)) \partial t\right]
$$

La procédure adoptée initialement par Kurosawa [10] pour déterminer les temps de libre parcours $\Delta t$, est de tirer au sort des nombres $r$ à distribution uniforme entre 0 et 1 [12], et de résoudre l'équation intégrale (3):

$$
\int_{0}^{\Delta t} \lambda(k(t)) \partial t=-\operatorname{Ln}(r)
$$

c)- Lorsqu'une interaction a lieu, nous déterminons sa nature par tirage au sort, et nous modifions, en conséquence, l'énergie et le vecteur d'onde de l'électron. Calculée par l'équation de Boltzmann, la répartition des électrons change; nous calculons alors le champ électrique qui en résulte à des intervalles de temps suffisamment petits, pour pouvoir le supposer constant entre deux calculs [11-13].

Nous considérons un modèle simplifié à trois vallées non paraboliques mais isotropes. Dans les vallées supérieures, nous calculons la masse des électrons par la relation [14]: $\alpha=\left(I-m^{*} / m 0\right) \times \mathcal{E}$, où $m 0$ est la masse de l'électron dans le vide, $\mathrm{m}^{*}$ sa masse effective, $\varepsilon$ son énergie et $\alpha$ le coefficient de non parabolicité de la vallée où il se trouve. Cette relation n'est pas exactement celle appliquée dans le cas du modèle complet ; mais elle est suffisante pour décrire le comportement électrique du composé.

La procédure générale d'exécution du logiciel est composée de quatre étapes essentielles:

- Lecture du fichier de données concernant les paramètres du matériau.

- Entrée des données telles que le nombre de particules simulées (Ns), le pas du temps, le pas d'énergie, le champ électrique appliqué, le temps maximum de simulation (Ts), etc - Exécution du logiciel.

- Fourniture de deux fichiers de sorties:

* Le premier fichier contient les valeurs des probabilités d'interactions.

* Le deuxième contient les valeurs des grandeurs moyennes (énergie, vitesse, ...). 


\section{Résultats et discussion}

\section{3-1. Interactions élastiques}

Ces interactions n'influent pas de façon essentielle sur le comportement de l'électron du fait qu'il n'est pas dévié de sa trajectoire. Elles sont néanmoins introduites pour obtenir une description aussi quantitative que possible, de la dynamique électronique.

\section{3-I-I. Interactions piézoélectriques}

Ces interactions, en fonction de l'énergie de l'électron, dans les trois vallées $\Gamma, X$ et $L$, sont illustrées à la Figure 1 . Elles augmentent légèrement dans les vallées supérieures, avec la masse effective des électrons, mais elles restent très faibles (de l'ordre de 1015). Elles atteignent rapidement la saturation (vers $0.1 \mathrm{eV}$ ). Ceci concorde bien avec la théorie qui prédit que leur probabilité est inversement proportionnelle à la racine carrée de l'énergie $\varepsilon$. Parfois, l'interaction piézoélectrique est confondue avec celle due au potentiel de déformation, dans le cas d'une interaction intravallée.

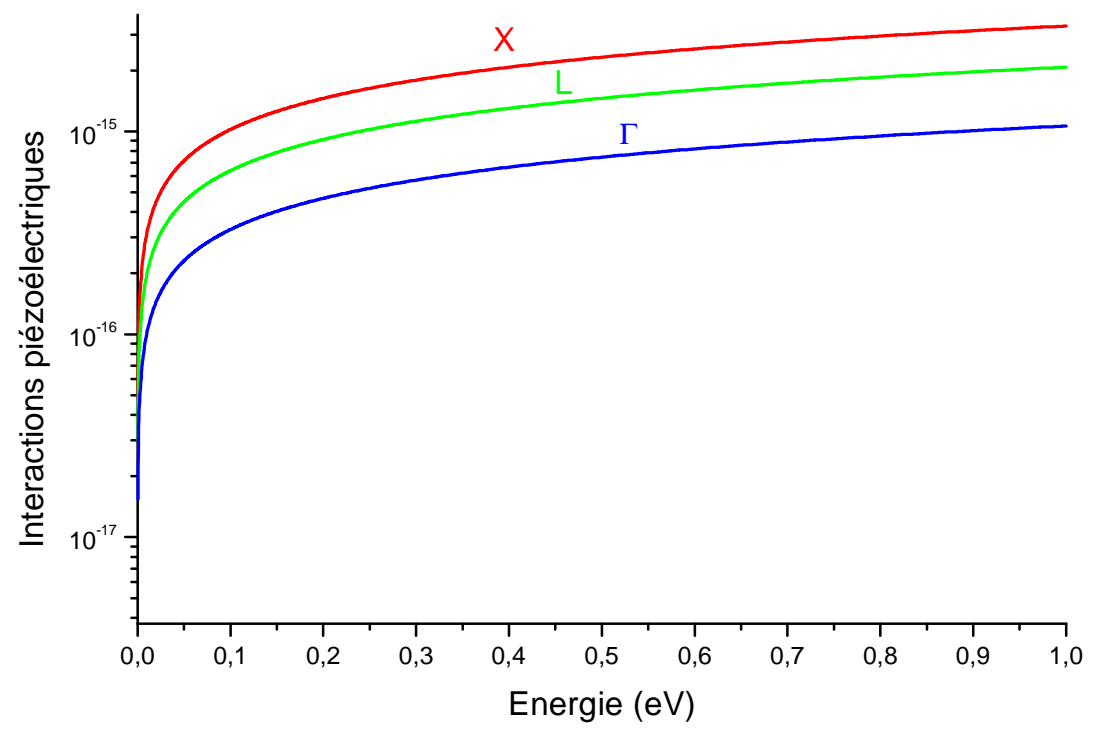

Figure 1 : Interactions piézoélectriques dans les trois vallées.

\section{3-1-2. Interactions acoustiques}

La Figure 2 illustre l'évolution de ces interactions en fonction de l'énergie, dans les trois vallées. 


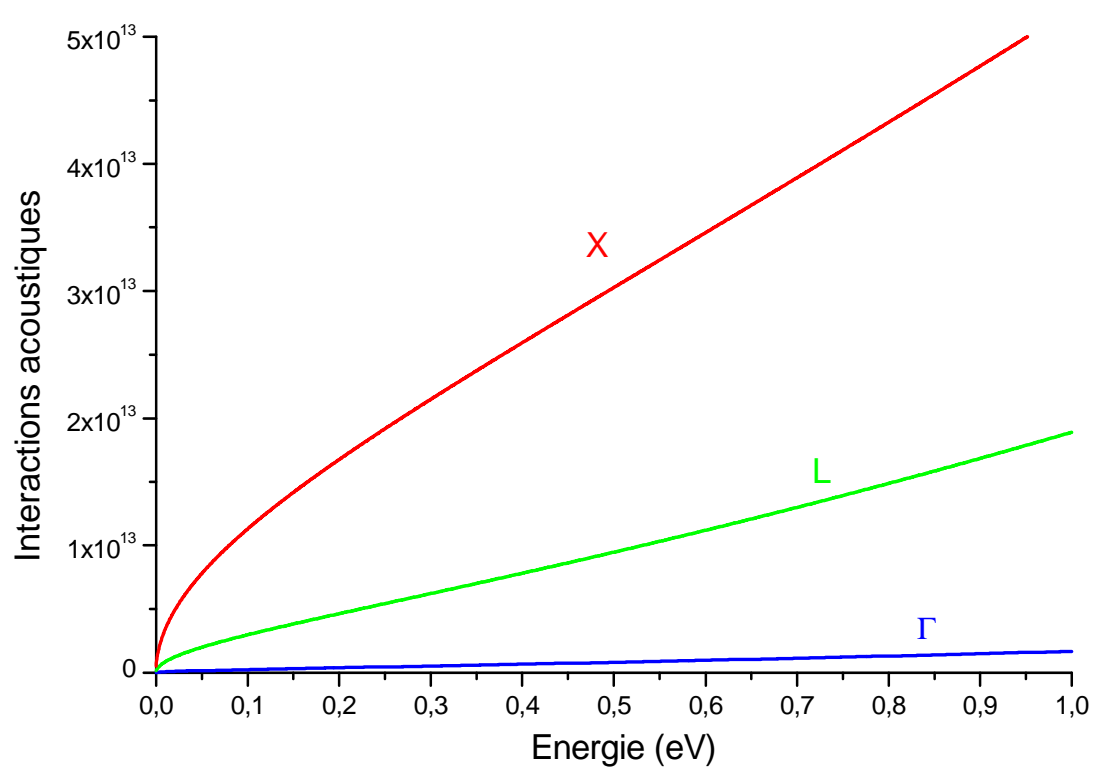

Figure 2 : Interactions acoustiques dans les trois vallées.

Ces interactions sont provoquées essentiellement par les effets du dopage en électrons. Dans la vallée $\Gamma$, ce type d'interactions est presque inexistant. Dans la vallée $L$, la probabilité augmente peu, et dans la vallée $X$, elle augmente considérablement avec l'énergie.

Pour des températures ou des champs très élevés, l'interaction acoustique devient alors prédominante, l'énergie d'électron est plus importante que celle d'un phonon optique (interaction intravallée). L'électron a ainsi tendance à transmettre au réseau une partie de l'énergie que lui a fournie le champ électrique. Ces interactions sont les plus dominantes des interactions élastiques.

\section{3-2. Interactions inélastiques}

Ces interactions sont dues essentiellement aux transitions intervallées qui permettent le transfert des porteurs, d'une vallée à une autre vallée équivalente ou non équivalente. Elles sont accompagnées d'absorption ou d'émission d'un phonon dont la valeur dépend de l'écart d'énergie entre la vallée de départ et la vallée d'arrivée.

Une interaction intervallée ne peut avoir lieu que si l'énergie après absorption ou émission du phonon, est supérieure à l'énergie du fond de la vallée d'accueil (les énergies étant repérées par rapport au bas de $\Gamma$ ). 


\section{3-2-1. Interactions intervallées non équivalentes}

Sur les Figures 3 et 4, nous représentons toutes les transitions possibles des vallées: $\Gamma-\mathrm{L}, \Gamma-\mathrm{X}, \mathrm{L}-\mathrm{X}, \mathrm{X}-\mathrm{L}, \mathrm{L}-\Gamma$ et $\mathrm{X}-\Gamma$ (absorptions et émissions).

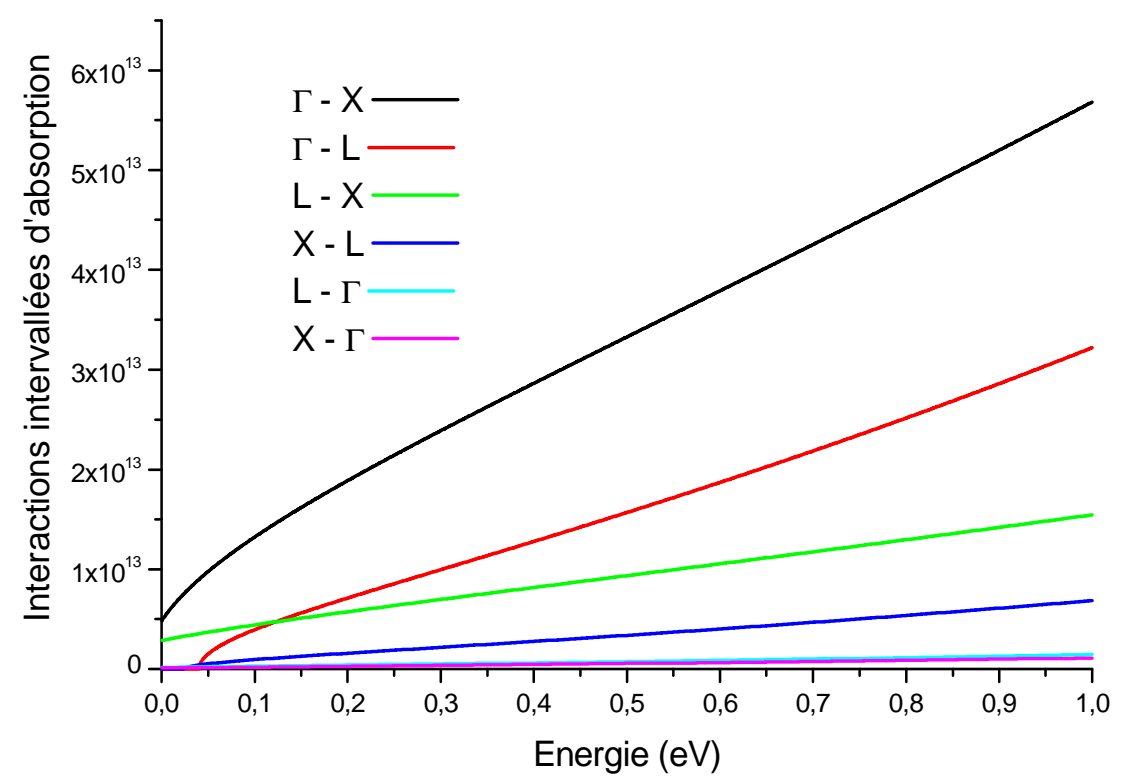

Figure 3 : Interactions intervallées d'absorption.

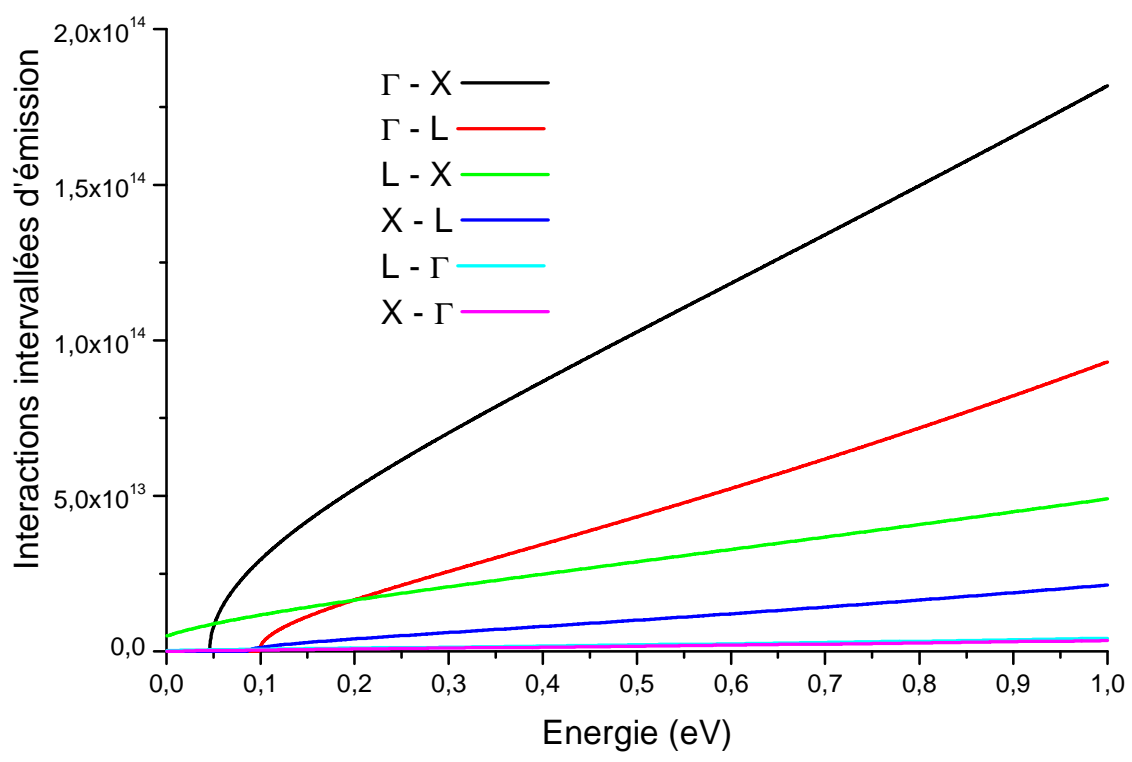

Figure 4 : Interactions intervallées d'émission 
Les interactions intervallées ne peuvent se produire que lorsque l'énergie des porteurs est suffisamment grande. Elles sont donc très peu probables à faible champ. Quand un électron est transféré vers une autre vallée, aux effets directs de l'interaction ellemême, viennent s'ajouter toutes les discontinuités dues à la non équivalence éventuelle des vallées d'origine et d'arrivée.

En particulier, comme l'énergie cinétique de l'électron se mesure à partir du minimum de la vallée qu'il occupe, un transfert vers une vallée non équivalente provoque une importante variation d'énergie cinétique correspondant à la différence de niveaux entre les deux vallées.

Les énergies des phonons émis ou absorbés sont comprises entre $8 \mathrm{meV}$ et $33.7 \mathrm{meV}$, donc très inférieures à l'énergie initiale des porteurs à température ambiante.

Nous tirons alors deux conclusions:

- Les probabilités de transition intervallée $\Gamma-X$ (absorptions comme émissions) sont les plus importantes.

- Les probabilités d'émissions sont plus grandes que celles d'absorptions.

\section{3-2-2. Interactions intravallées}

Dans la Figure 5, nous représentons les interactions intravallées en fonction de l'énergie des électrons.

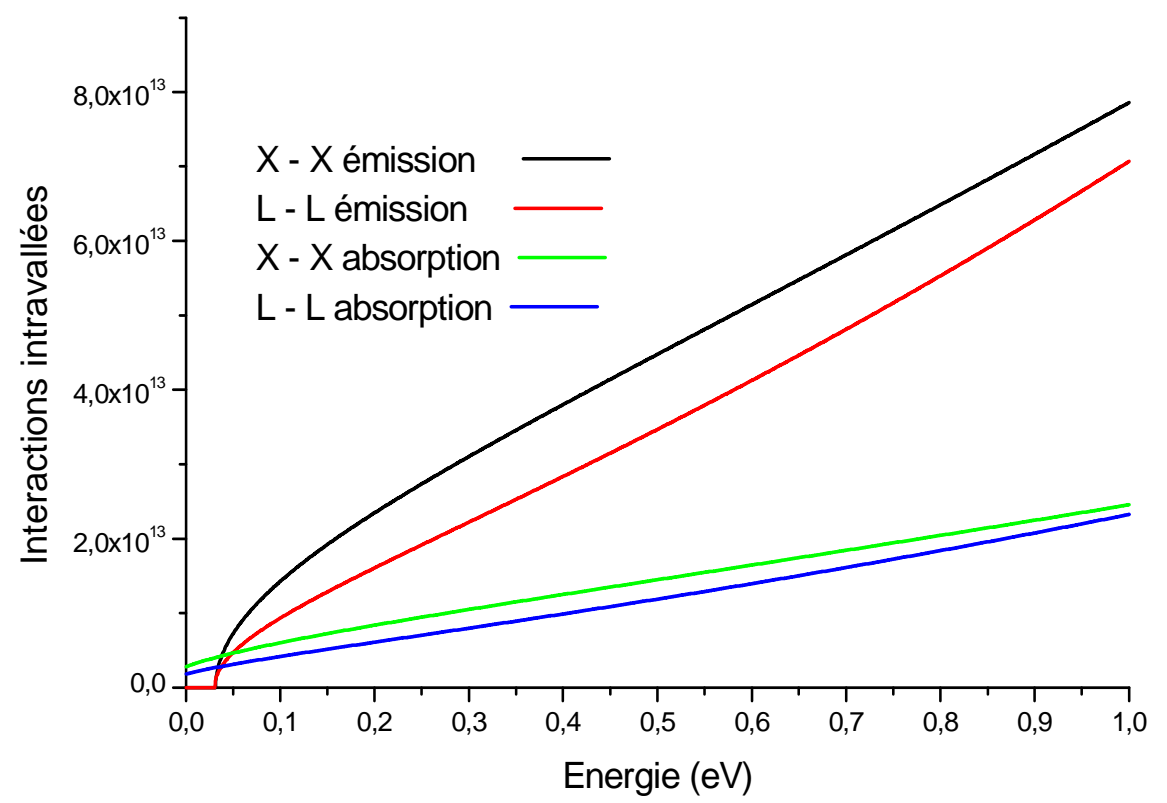

Figure 5 : Interactions intravallées. 
Comme pour les interactions précédentes, elles augmentent avec l'énergie des porteurs, et sont plus importantes dans les vallées supérieures, à cause de la masse effective. Là aussi, les probabilités d'émissions sont plus grandes que celles d'absorptions.

\section{3-2-3. Interactions optiques polaires}

La Figure 6 illustre l'évolution des interactions optiques polaires (absorptions et émissions) dans les vallées $\Gamma$, $L$ et $X$.

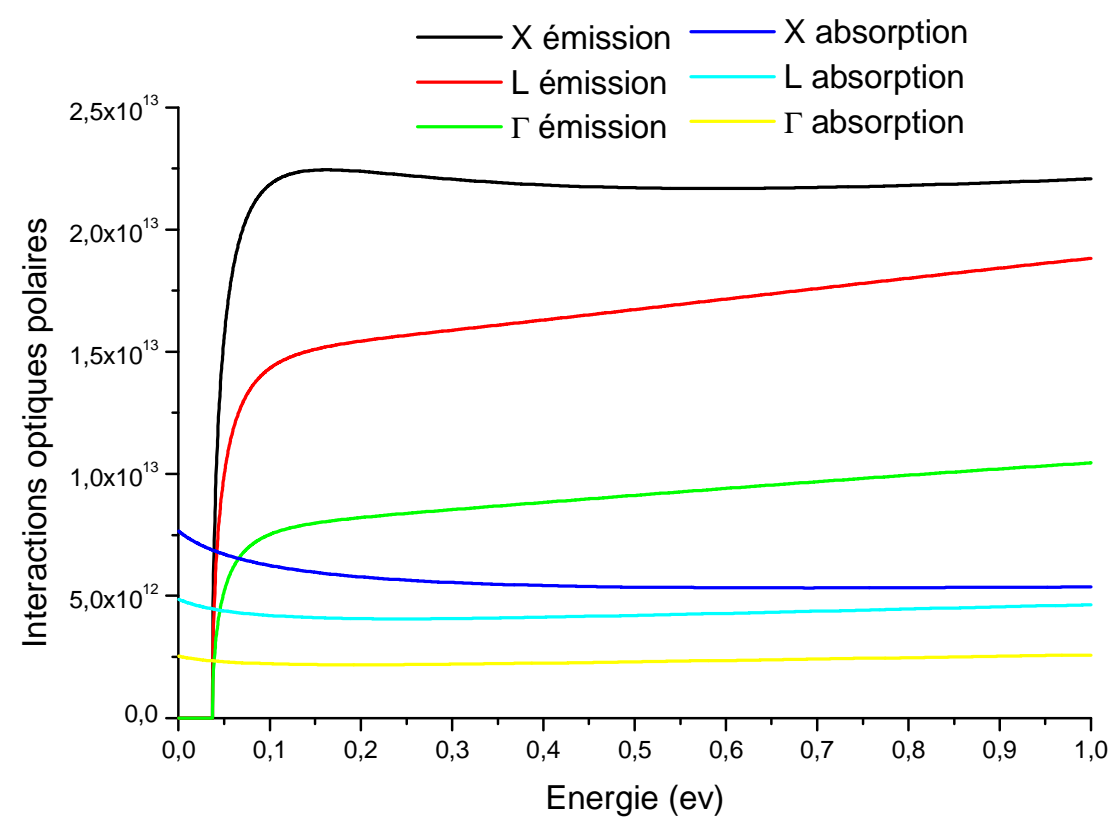

Figure 6 : Interactions optiques polaires.

L'interaction optique polaire prédomine en vallée centrale en champ faible. Elle est caractérisée par un échange de phonon optique polaire de l'ordre de $35 \mathrm{meV}$, et par une forte anisotropie sur l'ensemble des états finaux probables : les états correspondant à une déviation quasi nulle du vecteur d'onde, sont très fortement favorisés. L'électron, entraîné dans la direction du champ pendant la phase de vol libre, ne sera donc que détourné de cette direction sous l'effet de cette interaction. A faible énergie $(<0.5 \mathrm{eV})$, seules les interactions optiques polaires (émissions et absorptions) et les interactions acoustiques, influent sur le comportement des porteurs.

Tant que l'énergie est inférieure à $0.6 \mathrm{eV} \pm \Gamma \omega i$ aucun porteur ne peut être transféré dans la vallée supérieure $L$. Nous trouvons aussi que les interactions d'émissions sont plus importantes que celles d'absorptions. 


\section{3-3. Vitesse de dérive, dans le régime transitoire.}

\section{3-3-I. Vitesse de dérive Vd(t) dans la vallée centrale}

Dans la Figure 7 , nous représentons les vitesses de dérive en fonction du temps, à différents champs électriques. Il est à noter que le champ électrique, appliqué à $t=0$, reste constant pendant tout le temps d'observation.

Nous pouvons faire quatre constatations :

1. Les pics de survitesse apparaissent à partir de $10 \mathrm{kV} / \mathrm{cm}$.

2. Les pics de survitesse deviennent de plus en plus importants lorsque le champ électrique augmente.

3. Les pics se déplacent vers les temps faibles quand le champ augmente.

4. Le régime stationnaire est atteint plus vite à fort champ.

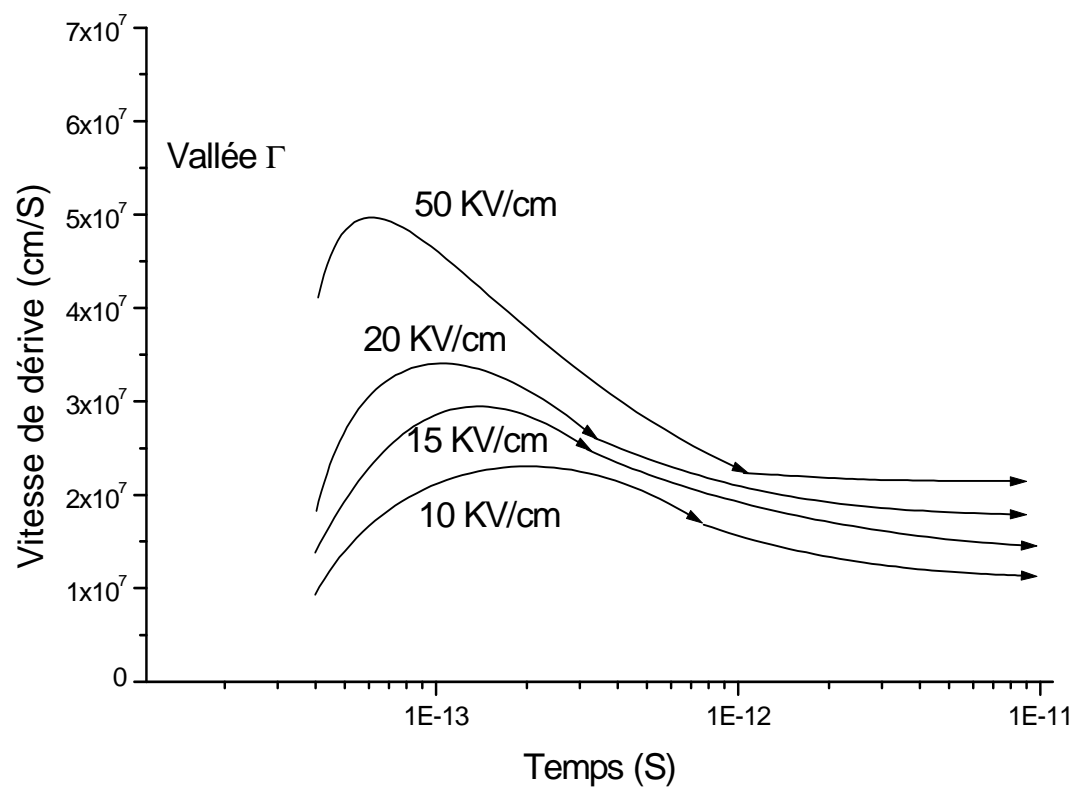

Figure 7 : Vitesse de dérive dans la vallée $\Gamma$, en régime transitoire.

\section{3-3-2. Vitesse de dérive $V d(t)$ dans les vallées $L$}

Sur la Figure 8, nous présentons les vitesses de dérive dans les vallées de symétrie L, pour différentes valeurs du champ électrique. Dans ces vallées, les masses des porteurs sont plus grandes que dans $\Gamma$, leurs vitesses y sont alors plus faibles. De plus, la 
contribution de ces vitesses, reste négligeable à faible champ. Ceci est dô au fait que ces vallées restent non peuplées, jusqu'au moment où les porteurs acquièrent une énergie assez importante pour qu'ils puissent être transférés dans $L$.

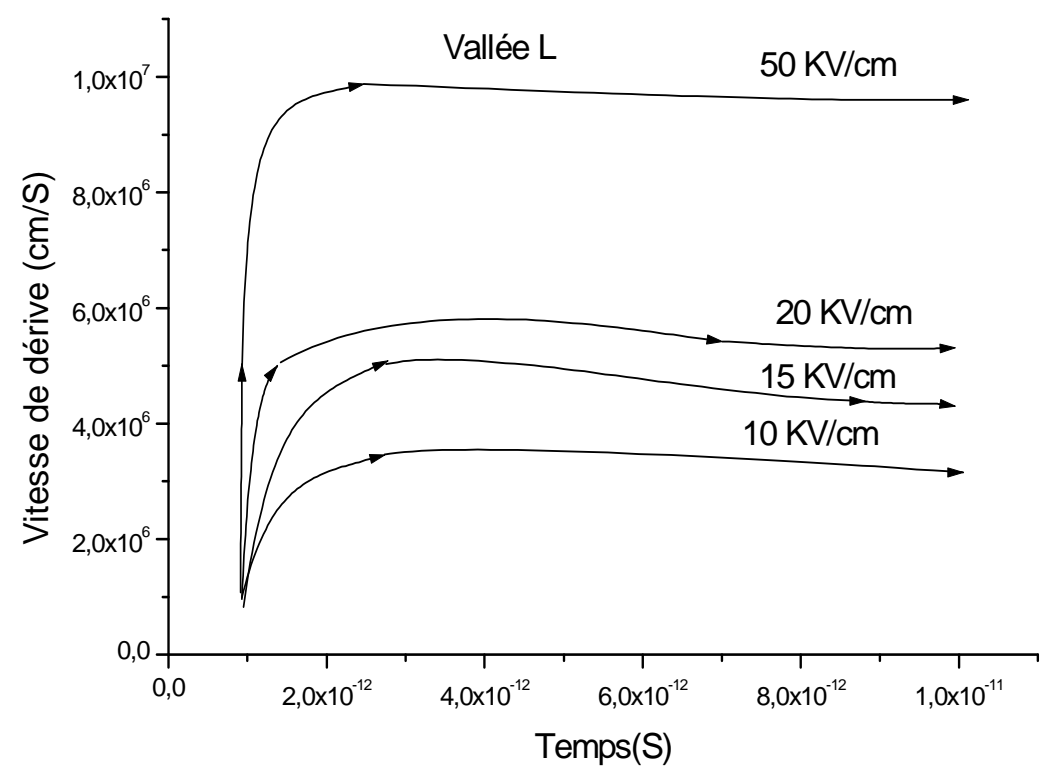

Figure 8 : Vitesse de dérive dans les vallées L, en régime transitoire

\section{3-3-3. Vitesse de dérive dans les vallées X}

Les vitesses de dérive dans les vallées de symétrie $X$, sont représentées à la Figure 9 . Dans les deux vallées $L$ et $X$, les vitesses restent nulles tant que l'énergie des porteurs reste inférieure à $0.8 \mathrm{eV}$, ce qui correspond à un temps de l'ordre de 1 ps. A partir de ce moment, elles augmentent presque instantanément. Dans la vallée $X$ : la vitesse est d'environ $0.1 \times 107 \mathrm{~cm} / \mathrm{s}$ pour un champ électrique de $10 \mathrm{kV} / \mathrm{cm}$, elle atteint à peine $0.4 \times 107 \mathrm{~cm} / \mathrm{s}$ pour $\mathrm{E}=50 \mathrm{kV} / \mathrm{cm}$.

Dans la vallée $\mathrm{L}$ : elle est de l'ordre de $0.3 \times 107 \mathrm{~cm} / \mathrm{s}$ pour $\mathrm{E}=10 \mathrm{kV} / \mathrm{cm}$, et elle atteint $1 \times 107 \mathrm{~cm} / \mathrm{s}$ pour $\mathrm{E}=50 \mathrm{kV} / \mathrm{cm}$.

Ces vallées restent non peuplées jusqu'à un champ de $50 \mathrm{kV} / \mathrm{cm}$. 


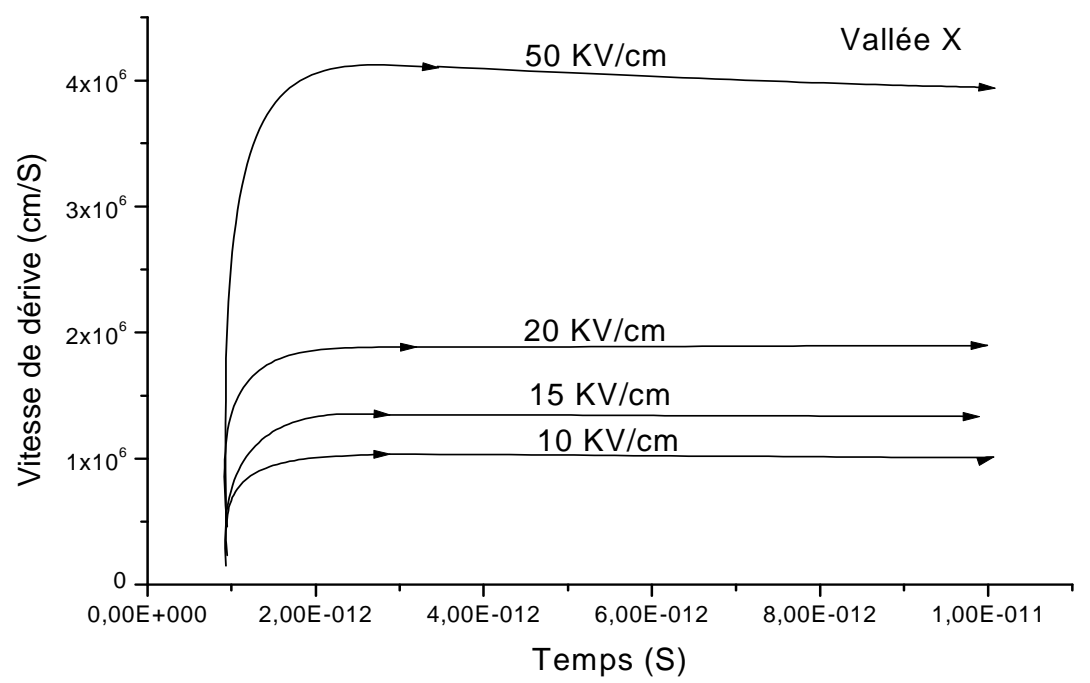

Figure 9 : Vitesse de dérive dans les vallées $X$, en régime transitoire

\section{3-4. Vitesse de dérive moyenne}

Les vitesses moyennes $\mathrm{Vd}(t)$, dans les trois types de vallées $\Gamma$, $L$ et $X$ (Figure 10), sont les mêmes pour les champs inférieurs à $10 \mathrm{kV} / \mathrm{cm}$.

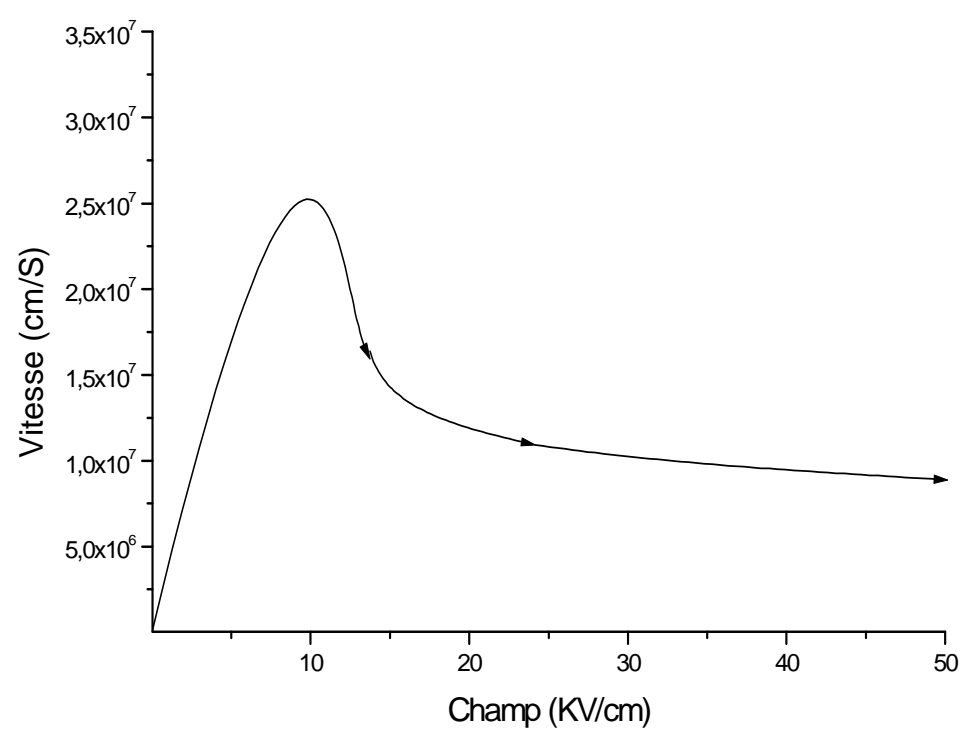

Figure 10 : Vitesse de dérive moyenne en fonction du champ électrique. 
- A faible champ ( $\mathrm{E}<0.6 \mathrm{kV} / \mathrm{cm}$ ) : La mobilité est importante; les électrons ont une énergie inférieure à $\hbar \omega_{0}$, et les interactions se font essentiellement avec les phonons acoustiques et / ou les impuretés.

- Pour $0.6 \mathrm{kV} / \mathrm{cm} \leq \mathrm{E} \leq 3 \mathrm{kV} / \mathrm{cm}$ : L'énergie des électrons est supérieure à $\hbar \omega_{0}$, mais elle est insuffisante pour que le transfert dans la vallée $L$ soit possible $(E<0.3 \mathrm{eV})$. Les interactions se font avec les phonons optiques polaires.

- Pour $3 \mathrm{kV} / \mathrm{cm} \leq \mathrm{E} \leq 10 \mathrm{kV} / \mathrm{cm}$ : L'énergie stationnaire atteint et dépasse l'énergie de transfert $\Delta \Gamma \mathrm{L}$, une proportion importante d'électrons se trouvent transférés dans la vallée $L$ de la bande de conduction. La masse effective des électrons dans cette vallée, a une valeur élevée $\left(0.2 \mathrm{~m}_{0}\right.$ au lieu de $0.06 \mathrm{~m}_{0}$ dans $\left.\Gamma\right)$, et la distribution des vitesses après transfert est quasi-isotrope ; ceci explique la décroissance de la vitesse.

- A partir de $10 \mathrm{kV} / \mathrm{cm}$ : la vallée $L$ est très peuplée, la vitesse moyenne des électrons décroît alors.

\section{3-5. Phénomène de survitesse}

Considérons un matériau libre de toute contrainte et non soumis à l'action d'un champ électrique. Les porteurs sont alors en équilibre thermique : $\langle v\rangle=0$ et $\langle\varepsilon\rangle=3 / 2$ KBT.

A l'instant $\uparrow=0$, nous appliquons un champ électrique $\vec{E}_{0}$. La courbe donnant la vitesse moyenne des électrons en fonction du temps, pour différents champs, est reportée sur la Figure 11.

La courbe comporte quatre zones. Pour le champ de $20 \mathrm{kV} / \mathrm{cm}$, par exemple :

- Dans la première zone : $\uparrow<\uparrow 0$ ( $+0=0.08$ ps), la vitesse croît linéairement avec le temps.

- Dans la deuxième zone : $\mathrm{t0}<\mathrm{t}<\mathrm{tl}(\mathrm{tl}=0.09$ ps), la vitesse continue de croître mais moins rapidement.

La vitesse moyenne passe alors par un maximum $(v=3.4 \times 107 \mathrm{~cm} . \mathrm{s}-\mathrm{l})$ très supérieure à la valeur stationnaire correspondant au champ $\mathrm{E} 0$ : c'est le phénomène de survitesse qui apparaît.

- Dans la troisième zone : $\mathrm{t}<\uparrow<\uparrow 2(\uparrow 2=2.0 \mathrm{ps}$ ), la vitesse décroît rapidement.

- Dans la quatrième zone : $\uparrow \geq \uparrow 2$, la vitesse est égale à la vitesse stationnaire correspondant au régime stationnaire.

Pour E0 $=50 \mathrm{kV} / \mathrm{cm}$, la vitesse atteint un pic de l'ordre de $6 \times 107 \mathrm{~cm} / \mathrm{s}$, à un temps plus petit. 


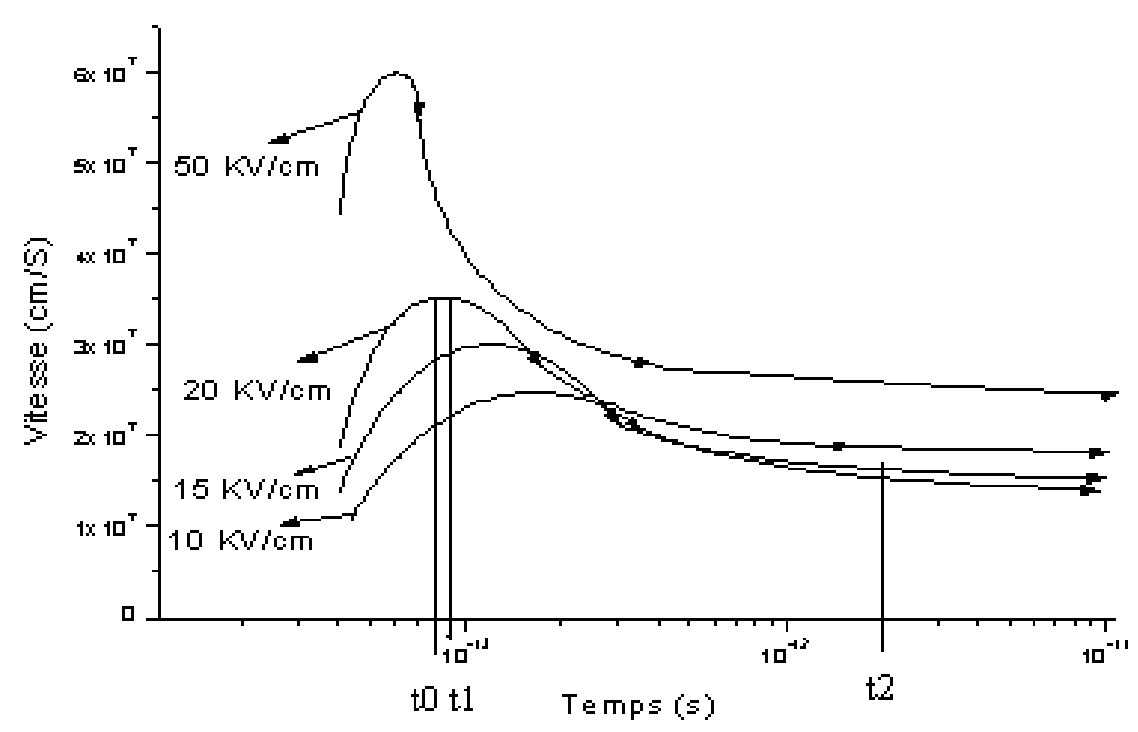

Figure 11 : Influence du champ électrique sur la vitesse moyenne.

\section{Conclusion}

Nous avons adapté la méthode de Monte Carlo écrite en langage Fortran, qui est précise, fiable et même parfois plus simple que les techniques numériques classiques. Néanmoins elle demande beaucoup d'espace mémoire, c'est l'une des principales difficultés, une station permettrait de simuler plus d'électrons, et ainsi obtenir de meilleurs résultats. Nous l'avons appliquée pour calculer les différentes interactions ainsi que le transport électrique dans le composé ternaire Al0.15Ga0.85As.

- Les interactions piézoélectriques sont négligeables dans les trois vallées.

- Les interactions acoustiques sont négligeables dans la vallée $\Gamma$, et plus importantes dans $X$ que dans $L$.

- Les interactions inélastiques d'émissions (intervallées, intravallées et optiques polaires) sont plus importantes que celles d'absorptions.

- Dans la vallée central, les pics de survitesse apparaissant à partir du champ critique, ils sont plus importants et surviennent plus rapidement quand l'échelon du champ augmente.

- Dans les vallées $L$ et $X$; les vitesses sont moins importantes que dans la vallée $\Gamma$, et elles atteignent le régime stationnaire vers $2 \mathrm{ps}$.

Ces résultats sont en accord avec les résultats numériques et expérimentaux édités.

La caractéristique d'émission trouve des applications dans le domaine technologique d'optoélectronique par exemple les Lasers à hétérojonction. 


\section{Références}

[1] - A. Negol, A. Guyot and J. Zimmermann, "A dedicated circuit for changed particles simulation using the Monte Carlo", Proceeding of IEEE International conference on application specific systems, architectures and processors (AASAP 97), IEEE (1997)

[2] - J. Zimmermann, "Etude par la méthode de Monte Carlo des phénomènes de transport électronique dans le Silicium de type $\mathrm{N}$ en régimes stationnaire et non stationnaire. Application à la simulation des composants submicroniques", Thèse, Lille 1 (1980)

[3] - Joseph Fourier, "Physique des matériaux", Thèse, Grenoble 1 (décembre 2004)

[4] - Benyounés BOUAZZA et al., "Etude du transport électronique dans le substrat InAs de type N par la simulation de Monte Carlo", Afrique Science, 01(1) (2005) 55-67, http://www.afriquescience.org

[5] - Jérôme SAINT-MARTIN, "Etude par simulation Monte Carlo d'architectures de MOSFET ultracourts à grille multiple sur SOl”, Thèse, Pris-Sud (décembre 2005).

[6] - T. Kurosawa, Journal of the physical society of Japan, supplement 21 (1966) 424

[7] - H. D. Rees, Journal of physics and Chemistry of solids, Vol 30 (1969) 643

[8] W. Fawcett, A. D. Roardman and S. Swain, Journal of Physics and Chemistry of solids, Vol 31 (1970) 1963

[9] J. G. Ruch and W. Fawcett, Journal of applied Physics, Vol 41 (1970) 3843

[10] - P. J. Price, IBM Journal of Research and Development, Vol 17 (1973) 39

[11] - S. Galden "Etude du transistor bipolaire à double hétérojonction Si/SiGe/Si par la simulation Monte Carlo", Thèse, Paris sud (1992).

[12] - O. Mouton, J. L. Thobel, and R. Fauquemberg "Monte Carlo simulation of high-field electron transport in GaAs using an analytical band structure model", J. Appl. Phys., 74 (10)(1993)

[13] - J. L.Thobel, "Simulation Monte Carlo du transport électronique et des phénomènes de diffusion dans les systèmes à base de semi-conducteurs III-V" Thèse d'habitation, Lille 1 (2000)

[14] - A. F. M. Anwar, Wu. Shangli and T. Richard, "Temperature dependent transport properties in GaN, AlxGal-xN, and InxGal-xN semiconductors", IEEE Transactions on Electron Devices, Vol.48, $\mathrm{N}^{\circ} 3$ (March 2001) 567-572 